\title{
Development of numerical modelling of analysis program for energy-dissipating behaviour of velocity dependent hydraulic damper
}

\author{
M-H SHIH ${ }^{1}$, W-P SUNG ${ }^{2, *}$ and M-J WU ${ }^{3}$ \\ ${ }^{1}$ Department of Civil Engineering, National Chi-Nan University, Pu-Li, Nan-Tou, \\ Taiwan \\ ${ }^{2}$ Department of Landscape Architecture, National Chin-Yi University of \\ Technology, Taichung, Taiwan \\ ${ }^{3}$ Department of Medicine, Taichung Veteran General Hospital, Chung Shan \\ Medical University, Taichung, Taiwan \\ e-mail: wps@ncut.edu.tw; drwpsung@yahoo.com.tw
}

MS received 3 June 2009; revised 17 August 2009; accepted 27 August 2010

\begin{abstract}
A relief valve parallel to the throttle valve is added to a Velocity dependent hydraulic damper (VDHD) so that the orifice size that regulates the oil flow can be adjusted. This device adjustment will allow the damper to have an adaptive control of damping by changing its damping coefficient. A mathematical model including a serial friction model and a small damper that is parallel to the friction model added to the Maxwell model for simulating the actual energy-dissipating behaviour of the VDHD was proposed in this research. To extend the useful value of VDHD, a numerical analysis model based on the SAP2000 nonlinear analysis program was applied to simulate the energy-dissipating characteristics of VDHD in this study. The analysis results obtained by using the mathematical model and the proposed SAP2000 numerical model conform to the seismic resistant test results, and confirm that the SAP2000 nonlinear analysis program can accurately describe the actual energy-dissipating behaviour of the VDHD installed on structures under various energy-dissipating situations.
\end{abstract}

Keywords. Throttle valve; relief valve; damping coefficient; application of modified Maxwell theory; simulated model for SAP2000.

\section{Introduction}

Several earthquakes with strong magnitude, such as the Chi-Chi Earthquake in Taiwan (1999), the earthquakes in Hokkaido and Russia (2003), Turkey and Sumatra (2004), Indonesia (2004, 2005), Iran (2005, 2006), Pakistan (2005, 2008), China (2008), and Italy and Japan (2009), seriously threatened the safety and property of the residents. Various types of structural control

${ }^{*}$ For correspondence 
technologies have been developed to solve the safety and functional problems for structures under the excitation of external force. These structural control techniques are usually applied for diminishing the vibration response of the structure under the excitation of earthquake or strong wind. Some of the structural control systems use the characteristics of oil viscosity to develop various types of hydraulic dampers for dissipating the dynamic energy. These oil-pressure type dampers have the advantages of compact size, low vibration, aptness of automation, and high durability. In recent years, numerous dampers including the oil-pressure components (Taylor 1992; Lee \& Taylor 2001; Hsu et al 2000; Kurata et al 1999; Kurata et al 2000; Dyke et al 1998; Xu et al 2000) have been proposed for minimizing structural vibrations. The damping force is related to the square of axial velocity, and the viscous damping ratio is related to the shape and the location of the orifice in the pistons. However, these oil-pressure dampers are passive dampers because their behaviours cannot be controlled and adjusted. Thus, (Shih et al 2002; Kurata et al 1999; Dyke et al 1998; Symans \& Constantinou 1997) have attempted to control the hydraulic pressure with additional oil-pressure valves in order to obtain an adaptive control by varying the damping coefficient of the damper.

Shih et al $(2002,2003)$ proposed new hydraulic dampers i.e. displacement dependent semiactive hydraulic damper (DSHD), and accumulated semi-active hydraulic damper (ASHD). Both devices have a relief valve parallel to the throttle valve for adjusting the damping coefficient by varying the orifice area to adjust the oil pressure. Thus, the mechanical behaviours of these two dampers can be regulated for gaining adaptive control of the dampers. Then, (Shih \& Sung 2004, 2006 and Shih \& Sung 2006) proposed a new controllable energy dissipation device, i.e. velocity and displacement dependent hydraulic damper (VDHD); it includes a type of controllable energy-dissipating element installed in the passive control system. This proposed damper consists of hydraulic jack, check valve, relief valve and throttle valve, as shown in figure 1 . The energy dissipation characteristics of the proposed damper are controlled by regulating the flow of oil in the hydraulic jack. Results of laboratory studies demonstrate that the development of damping force is affected by the velocity of piston, and a limiting relief pressure of relief valve. When the oil pressure is smaller than a default value, the relief valve will be completely closed. If the pressure is equal to or slightly greater than the default value, the relief valve will open up. The size of the aperture is related to the overloading pressure for keeping the internal pressure close to a constant value (Symans \& Constantinou 1997). Therefore, if the internal pressure of the damper keeps expanding or contracting to approach the default pressure of the relief valve, the damper's transformational and frictional behaviours are equivalent. When the VDHD device performs at very high piston velocity, its energy dissipation behaviour is similar to the yielding behaviour of metals. Therefore, Shih et al (2007) proposed a discrete solution to modify the Maxwell theory for simulating the energy-dissipating behaviour of this damper.

The analytical model, such as the Maxwell model that simulates the mechanical properties of a hydraulic damper, consists of a series of dashpots and springs for analysing and simulating the energy dissipation behaviour of a damper such as those proposed in this study. Makris \& Constantinou (1991) proposed fractional derivative Maxwell models to represent the energy dissipation behaviour of fluid dampers. Later, Makris \& Constantinou (1993) used complex order derivatives to extend the fractional derivative Maxwell models. Makris et al (1995) used the complex-derivative Maxwell constitutive model to represent a particular polybutane fluid over broad frequency and temperature ranges. Some researchers (Constantinou et al 1998; Inaudi \& Hayen 1995; Patten et al 1996; Iwan \& Wang 1998) have considered the dynamic characteristics associated with the hydraulic damper by using the Maxwell model. Recently, the Maxwell type regularization has been extended to rate-dependent 


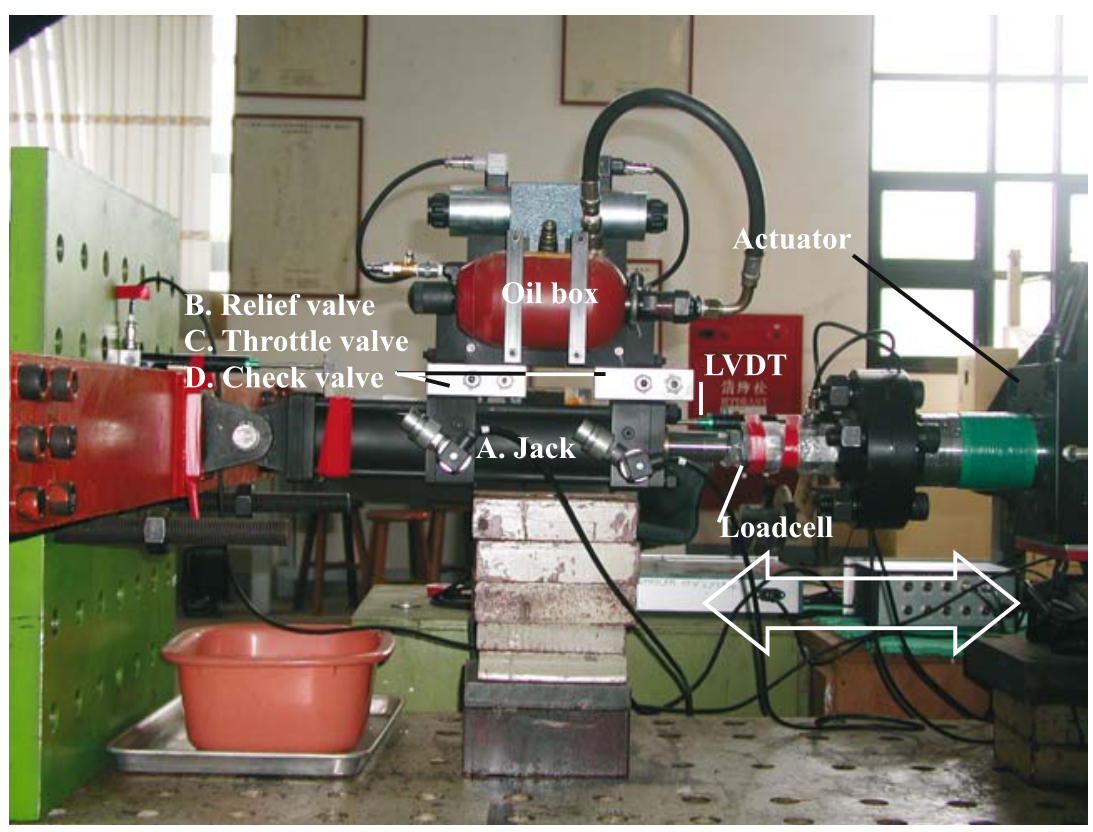

Figure 1. Experimental set-up.

regularization (Yang et al 2000; Liu et al 2000; Laursen \& Oancea 1997) with a nonlinear dashpot (Sivaselvan \& Reinhorm 2000). Besides, the SAP2000 nonlinear analysis program (SAP2000, 2002) is a state-of-the-art analytical method for a simple small 2D static frame, or a large complex 3D nonlinear dynamic analyses. The SAP2000 program is widely used as a design tool by engineers for the structural design of buildings, bridges, factories, industrial and public works, and other facilities. Extending the capacity of the analytical model, which consists of the analytical elements of SAP2000 nonlinear analysis program is proposed to simulate the seismic resistant property of the VDHD damper. This is done by connecting the friction model in series with the Maxwell model for investigating functions of the VDHD, and with a numerical model for calculating the damping force. The numerical results of this proposed analytical model based on SAP2000 are compared to verify the practical application of these proposed models for simulating the energy-dissipating behaviours of structure equipped with VDHD, and confirming the similarity between the experimental and the calculated results.

\section{Elements of velocity dependent hydraulic damper, VDHD}

The passive energy-dissipating behaviour of the velocity dependent hydraulic damper (VDHD) as proposed in this research is mainly generated by using two valves contained in the damper, i.e. the relief valve and the throttle valve. The former can limit the maximum pressure of the hydraulic cylinder whereas the latter is connected in parallel with the relief oil path. The throttle valve has a small circulating hole that can be adjusted externally and manually. When viscous oil is flowing in the hole, its pressure should overcome the pressure drop that is normally proportional to the first and second exponential of its flow velocity. 


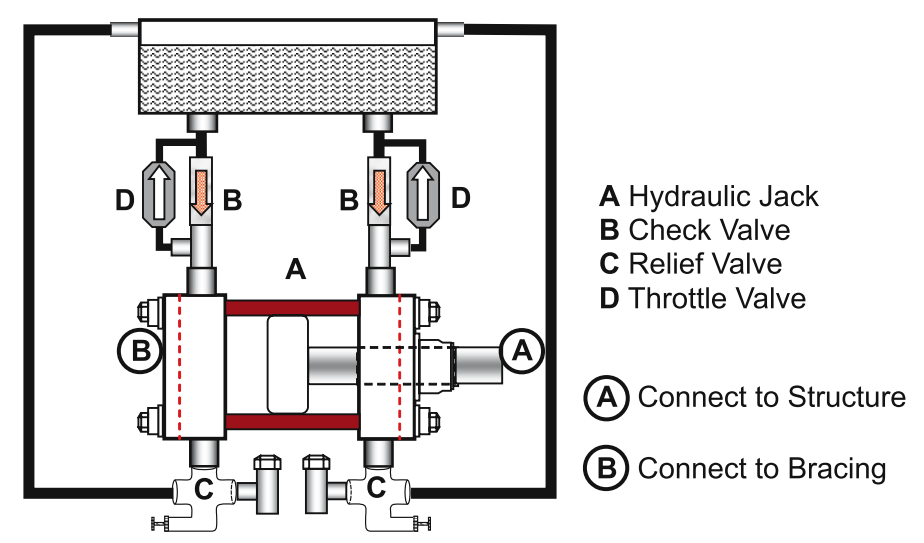

Figure 2. The structure of VDHD.

The objective of installing this valve in parallel is to allow the damper to return to its original position after structural vibration. However, this devise cannot be considered as a reliable control of the damper for generating a velocity-dependent damping force. The structure of VDHD is shown on figure 2. The structure with VDHD installation is shown on figure 3 . The following is a brief introduction to its main functions:

A. Hydraulic jack: The hydraulic jack, which is the main force transmission component of the damper, has two connecting joints. One joint is hinged to the structural body and the other joint is hinged with the bracing. The action force of the hydraulic jack equals the product of piston area and difference of the pressures exerted on either side of the piston.

B. Relief valve: The relief valve can maintain a certain pre-set pressure of the oil loop. If the pre-set pressure is exceeded, this valve will automatically open to release oil to reduce the pressure.

C. Throttle valve: This valve can control the passing oil flow rate by adjusting the crosssection of the oil pressure oil channel.

D. Check valve: The check valve can keep the oil flowing only in one direction to prevent it from flowing backwards.

\section{Energy-dissipating behaviour of VDHD}

The objective of these tests is to investigate the seismic resistant feasibility of VDHD. In these tests, the energy-dissipating behaviours of the various VDHD's, i.e. the passive VDHD using small and medium throttle valves connected in parallel, are compared by applying vibrations of constant amplitude but with various frequencies. Figure 1 shows the experimental set-up used to conduct the component test for investigating the energy-dissipating behaviours of VDHD that has been added with the relief and the throttle valves.

During the test, the orifice size of throttle valve is adjusted independently in order to conduct cyclic tests under various frequencies with $25 \mathrm{~mm}$ amplitude. The displacement capacity and the force of the damper are recorded; the hysteretic loops shown in figures 4 and 5 reveal 


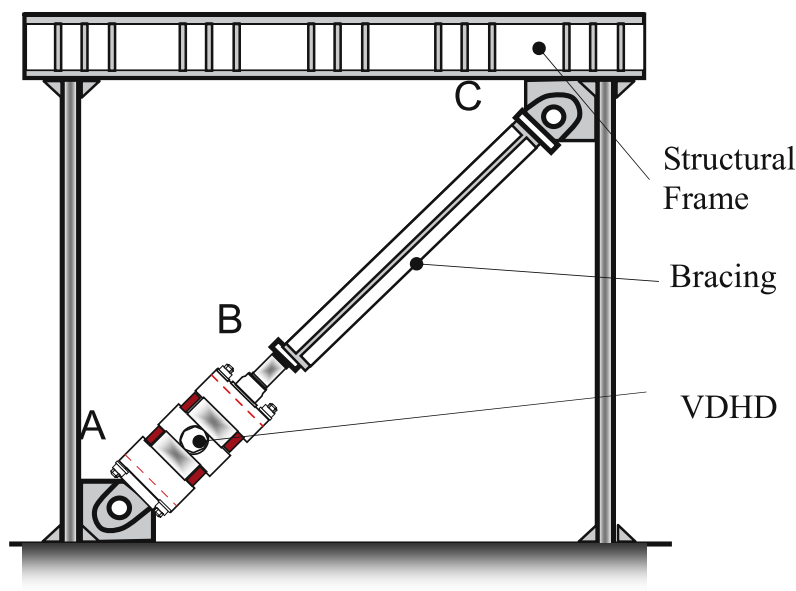

Figure 3. The assemblage of VDHD and structural frame.

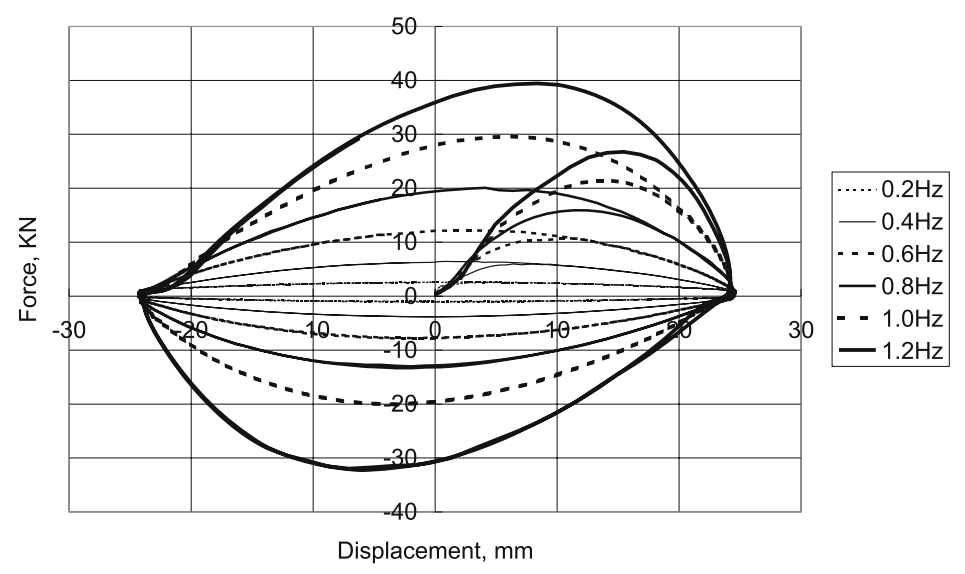

Figure 4. The tests of energy-dissipating behaviour of VDHD parallel with small Throttle valve.

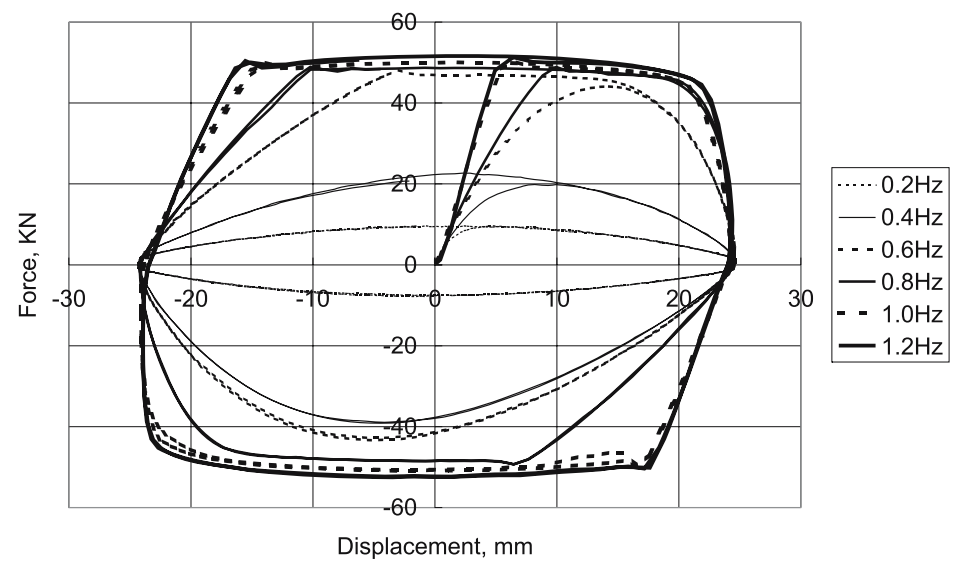

Figure 5. The tests of energy-dissipating behaviour of VDHD parallel with medium Throttle valve. 


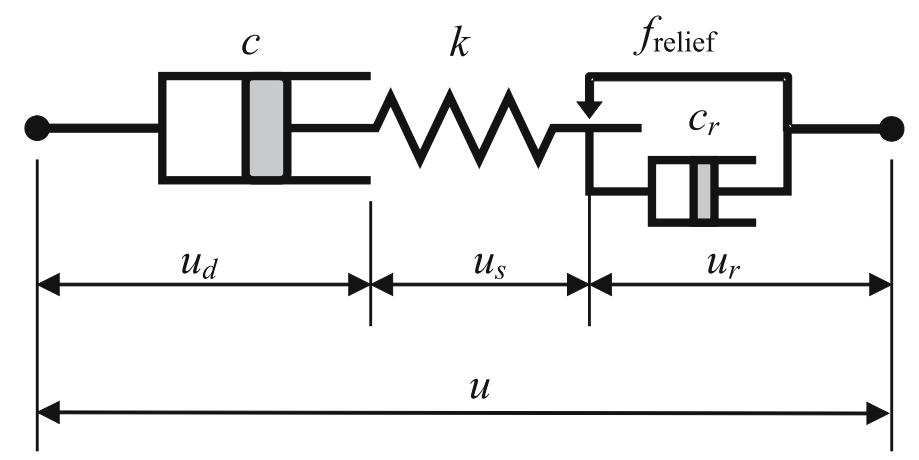

Figure 6. The Maxwell model with limitation of the relief pressure (Patten et al 1996).

the stable energy-dissipating performance of this hydraulic damper. The throttle valve that is adjusted externally and manually can easily change the relationship between the damping force and oil flow velocity. In addition, the function of relief valve is like a fuse in an electrical circuit. When the damping force is increased to an expected value, the relief valve will prevent the damping force from increasing further to protect other elements in this control system.

\section{Mathematical analysis model}

The component test results on the energy dissipation behaviour of VDHD show that these behaviours approach the energy dissipation behaviour of viscous dashpot models and elasticperfectly plastic models. Therefore, the Maxwell model is quoted and modified for simulating VDHD to demonstrate how the modified model can be applied for simulating the actual energy dissipation behaviour of VDHD accurately. Maxwell model, shown in figure 6 (Sung et al 2007), is quoted to derive the initial internal force of element, initial velocity, and internal force of VDHD at closing velocity to affect the internal force of the VDHD components.

The aperture of the relief valve is not fixed so that strictly speaking, the damping coefficient is not a constant value. All detailed interference of discrete solution to modify the Maxwell theory for simulating the energy-dissipating behaviour of this damper has been presented in literature (Sung et al 2007). The damping force is briefly expressed as follows:

$$
F=f_{\text {relief }}+c_{r} \dot{u}_{r}
$$

where $F$ : damper force; $f_{\text {relief }}$ : relief pressure of damper, unit: $k N ; c_{r}:$ damping coefficient of relief valve; $\dot{u}_{r}$ : deformation rate of elongation of relief valve model, unit: $\mathrm{m} / \mathrm{sec}$.

If a relief of the damper does not occur, the internal force of the element is:

$$
\dot{u}=\dot{u}_{d}+\dot{u}_{s}+\dot{u}_{r}
$$

where $\dot{u}_{d}$ : deformation rate of elongation of viscous damper, $\dot{u}_{d}=F / c$, unit: $\mathrm{m} / \mathrm{sec}$; $\dot{u}_{s}$ : deformation rate of elongation of spring, $\dot{u}_{s}=\dot{F} / k$, unit: $\mathrm{m} / \mathrm{sec}$. 
Solving the continuous solution and discrete solution, the system constants can be obtained as follows:

$$
\begin{aligned}
& \bar{D}=e^{-\frac{\bar{k}}{\bar{c}} \Delta T} \\
& \bar{E}=(1-\bar{D})\left(1+\frac{\bar{c}^{2}}{\bar{k} \Delta T}\right)-\bar{c}, \\
& \bar{G}=-(1-\bar{D}) \frac{\bar{c}^{2}}{\bar{k} \Delta T}+\bar{c},
\end{aligned}
$$

where

$$
\bar{k}=k ; \quad \bar{c}=\frac{c c_{r}}{c+c_{r}} ; \dot{\bar{u}}=\dot{u}+\frac{f_{\text {relief }}}{c_{r}} ;
$$

$k$ : stiffness; $c$ : damping coefficient; $\dot{u}$ : velocity across the Maxwell model.

The discrete solution is shown as follows:

$$
F_{1}=\bar{D} F_{0}+\bar{E} \dot{\bar{u}}_{0}+\bar{G} \dot{\bar{u}}_{1} \text {. }
$$

The internal force of component for the damper without relief is:

$$
F_{1}=D F_{0}+E \dot{u}_{0}+G \dot{u}_{1},
$$

where $D$ : initial coefficient of discrete solution, $D=e^{-\frac{k}{c} \Delta T} ; E$ : initial velocity coefficient of discrete solution, $E=(1-D)\left(1+\frac{c^{2}}{k \Delta T}\right)-c ; G$ : end velocity coefficient of discrete solution, $G=-(1-D) \frac{c^{2}}{k \Delta T}+c$.

Equation (6) is a discrete solution with the relief, and equation (7) is suitable for the discrete solution without the relief. The practical analytical procedures are as follows:

(i) If $a b s\left(F_{0}\right) \leq f_{\text {relief }}$, the equation (7) is applied to evaluate the damping force $F_{1}$ of next step;

(ii) If $a b s\left(F_{0}\right)>f_{\text {relief }}$, the equation (6) is applied to evaluate the damping force $F_{1}$ of next step.

\section{Numerical analysis method for SAP2000}

A mixed method combining the serial and the parallel connections for simulating the components of spring, viscous dashpot with friction element is applied to construct the physical model of VDHD. The mathematical model and discrete numerical solutions are developed based on this physical model. Although the discrete numerical solution is a convenient numerical analysis tool, the dynamic analysis program needs to be developed for energy design in accordance with mechanical properties of the physical VDHD. Most engineers do not have sufficient experience for developing this type of computer program. Therefore, the commercial software-SAP2000 Nonlinear Analysis Program is used to simulate the energy-dissipating behaviour of VDHD for extending its capacity.

\subsection{The element usage of SAP2000}

The proposed mathematical model is composed of elements for simulating popular components such as spring, dashpot and friction element. The following elements that are 


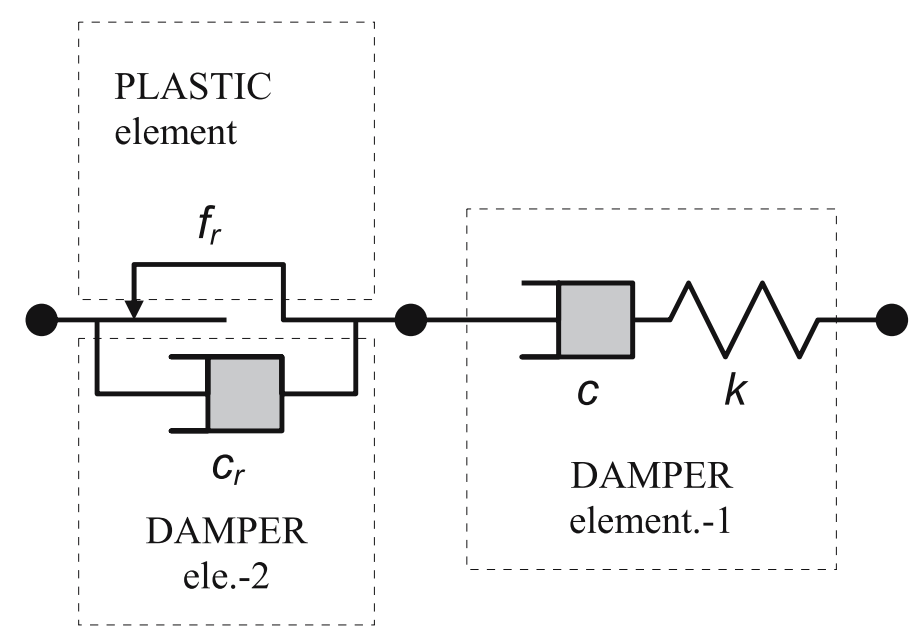

Figure 7. The physical model of VDHD.

available in the nonlinear link of SAP2000 are also used to construct the essential components of VDHD.

A. Damper element: The basis of the damper element in SAP2000 is a serial connection of spring element and dashpot element. Because the damping force of dashpot is a nonlinear function of velocity, this damper element can be used to simultaneously describe the physical model of VDHD that is equipped with a spring connected in series with the main damper as shown in figure 7. In addition, this damper can also simulate the auxiliary dashpot that is connected in parallel with the friction element. The stiffness of this element should be set comparatively large in order to simulate the behaviour of a pure dashpot.

B. plasticl element: If the plastic stiffness of a bilinear elastic-plastic element is set to zero, and the elastic stiffness is infinitely large, the mechanical behaviour of this element is similar to the friction element behaviour of an elastic stiffness, which is assumed to be a relatively large value as shown in figure 6 . The elastic stiffness is defined by setting the quantity of elastic deformation before it slides $0.1 \mathrm{~mm}$ or more.

\subsection{Analysis model of SAP2000}

The main difficulty of using SAP2000 to simulate repeatedly loading tests is the requirement that the time history of displacement records from SAP2000 should agree with the records of experimental data. Hence, the time history analysis method that is generally implemented to simulate this energy-dissipating behaviour of VDHD is applied for using the SAP2000 program to carry out the simulation. But, the mutuality of experimental data and simulated data obtained using SAP2000 should be examined using the trial-and-error method because the external force is unknown. Therefore, this analysis procedure using the trial-and-error method is time-consuming, and the simulated results hardly converge on the experimental data. The black box (figure 8) that stands for the element of VDHD in the original component test can be described by a single-degree-of-freedom system under the repeated action of expansion and contraction exerted by the hydraulic actuator (shown in figure 8a). The mass 
(a)

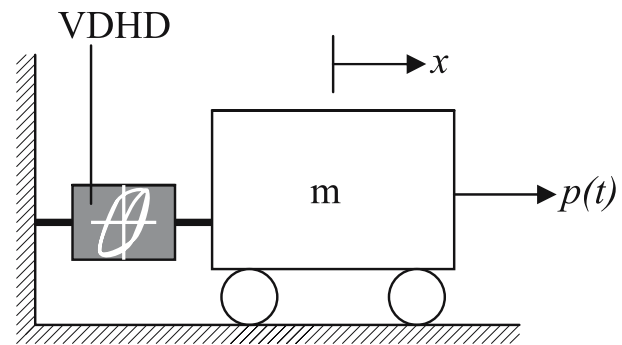

Auxiliary spring,

(b)

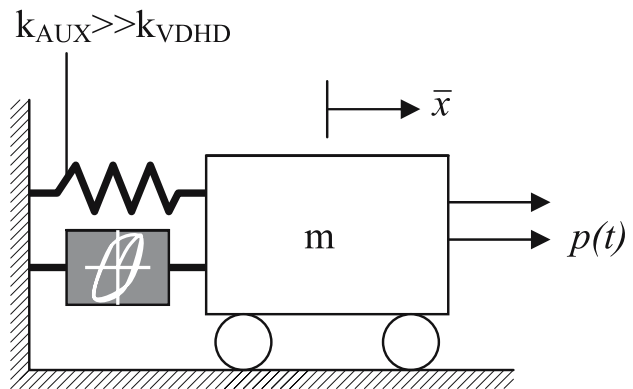

Figure 8. Analysis model for simulating component test of VDHD. (a) The typical analysis model. (b) The proposed analysis model for SAP2000.

in figure $8 \mathrm{~b}$ represents the equivalent mass; its value is generally not large so that it does not affect the subsequent analyses.

The function of the external force, $p(t)$ as shown in figure 8 , should be acquired by using the trial-and-error method because the external force is unknown before the analysis. The calculated displacement function $x(t)$ is compared with the experimental data for checking the convergence. The equation of motion is expressed as:

$$
m \ddot{x}(t)+f_{\mathrm{VDHD}}(t)=p(t) .
$$

Using the spring with a large stiffness will cause the majority of reaction forces to conform to the mass block movement. Combining this type of analysis model with an auxiliary elastic spring is proposed in this research as shown in figure $8 \mathrm{~b}$. The auxiliary external force $\bar{p}(t)$ is defined as:

$$
\bar{p}(t)=k_{\mathrm{AUX}} \cdot x_{m}(t),
$$

where $x_{m}(t)$ is the time history of measured displacement of component test.

Therefore, the new equation of motion can be obtained as follows:

$$
m \ddot{\ddot{x}}(t)+f_{\mathrm{VDHD}}(t)+k_{\mathrm{AUX}} \bar{x}(t)=p(t)+\bar{p}(t) .
$$

Accordingly, when $k_{\mathrm{AUX}}$ is 3 to 4 orders of magnitude greater than VDHD, $p(t)$ can be ignored so that only the $\bar{p}(t)$ term remains in the equation. Therefore, either Eq. (9) or Eq. (10) can be used for calculating the displacement response $\bar{x}(t)$ when the external force term is the only auxiliary external force. Furthermore, the solution of equation (10) becomes a static equilibrium equation because only the auxiliary external force can be obtained as follows:

$$
\bar{x}(t)=x_{m}(t) .
$$




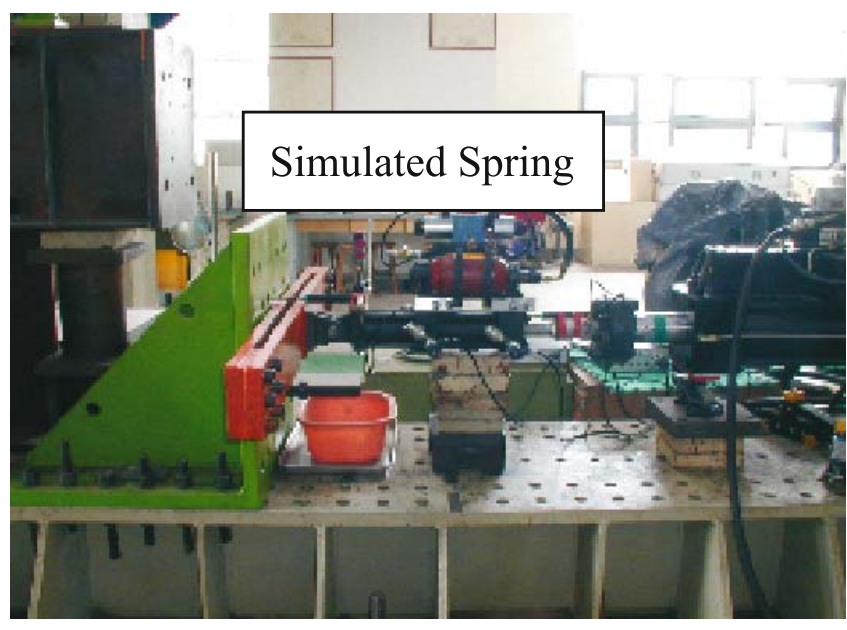

Figure 9. The simulated spring for bracing element.

Consequently, the objective displacement function is multiplied by the auxiliary spring with a large stiffness to yield an external force that will approximate the time history of VDHD responses. Hence, this method will make the tedious trial-and-error method more efficient to yield results faster when the experimental results of VDHD nonlinear component are compared.

\section{Test and verification of VDHD}

The seismic resistant tests of the VDHD system including the soft spring for simulating bracing are used to test and verify the accuracy of the proposed analysis model as shown in figures 1 and 9, the reality of the above numerical analysis model, and the efficiency of energy dissipation of this proposed damper.

\subsection{Experimental assemblage of VDHD}

The deformation behaviour of the bracing in an actual building, the inter-function between the expansion, and the contraction behaviour of the damper are important factors affecting the earthquake proof feasibility of VDHD. Therefore, in this seismic resistant test, a soft spring is utilized to simulate its the bracing element. The set-up for testing the VDHD seismic resistant and energy-dissipating performance is shown in figure 9. The whole test system contains a MTS100 actuator, which includes a Temposonic displacement gauge with Load Cell, a MTS407 controller, a control computer, a signal receiver, a UPS (unceasing power system) device, a test frame, and the VDHD. The overflow pressure of the relief valve is set at 130 bar to generate $50 \mathrm{kN}$ in VDHD without increasing the endured force capacity. The flowchart of signal measurement is shown in figure 10.

\subsection{Verification analysis}

The mathematical model presented in this paper is based on the serial and parallel connections of the linear viscous dampers and elastic plastic springs to simulate the energy-dissipating behaviour of VDHD. In addition, solutions of the governing equation are utilized to infer 


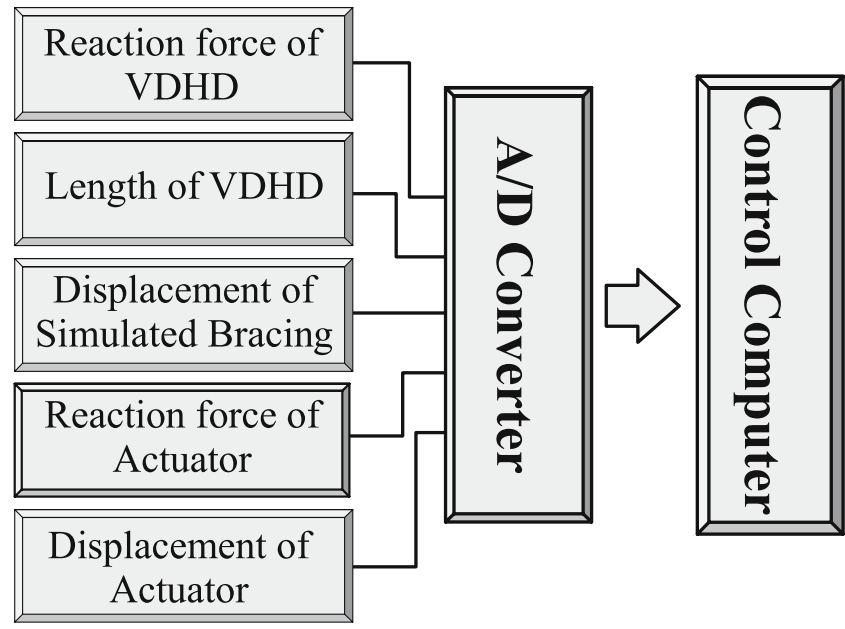

Figure 10. Flow chart of response and control signals.

discrete solutions; they are applicable to fast dynamic analyses. In order to simulate the real energy-dissipating behaviour of VDHD properly with this proposed mathematical model, some assumptions are made on various functions of VDHD as follows: (i) the viscous damper ( $c_{1}$ and $c_{2}$ damper) is linear, (ii) the elastic coefficient of damper $\left(k_{1}\right.$ and $\left.k_{2}\right)$ is constant, (iii) the damping coefficient of relief valve $\left(c_{r 1}\right.$ and $\left.c_{r 2}\right)$ is constant, and (iv) The relief force of oil cylinder is $\left(f_{y 1}\right.$ and $\left.f_{y 2}\right)$ constant.

These tests utilize the amplitude of $20 \mathrm{~mm}$ and frequencies varying from $0 \cdot 1$ to $2 \cdot 0 \mathrm{~Hz}$ frequency to evaluate whether the proposed mathematical and numerical model is appropriate for simulating the actual behaviour of the VDHD. The objective of these tests is to evaluate the force-displacement of this damper. Additionally, the comparison is conducted based on the mathematical and numerical model developed in this paper for verifying the accuracy of various energy-dissipating behaviours of this damper.

\section{Experimental, numerical analysis results and discussion}

\subsection{Experimental and numerical analysis results}

The proposed analysis model based on SAP2000N has undergone several tests and numerical simulations to verify its accuracy. The component parameters, identified from each hysteretic loop, are acquired to properly simulate all energy-dissipating behaviour with various frequencies as shown in table 1 . Figure 11 indicates that the model is suitable for simulating the repeatedly loading test using SAP2000 in this paper. As shown in table 2 that lists the element parameters, the restraint conditions of node 1,4 and 6 are set to be fixed whereas the constraint conditions for node 2 and 5 and for node 3 and 7 are set to be equal. The time history function is defined as time history of displacement of experimental records in the process of analysis, and the time history of loading function $\bar{p}(t)$ is defined as the mathematical product of the time history function and the stiffness of element (4).

Then, results of numerical analyses using the mathematical model and the simulated results obtained using SAP2000N are compared with the experimental data acquired using the seismic resistant test to verify the practical capability of these proposed models. The forcedisplacement relationships are shown in figure 12a to figure 12f. Figure 12a through figure 12d 
Table 1. The parameters identification results of VDHD component.

\begin{tabular}{lcc}
\hline Parameters items & Results & Units \\
\hline Main damping coefficient, $\boldsymbol{c}$ & 1.98 & $\frac{\mathrm{kN}}{\mathrm{mm} / \mathrm{sec}}$ \\
Spring stiffness, $\boldsymbol{k}$ & 14 & $\frac{\mathrm{kN}}{\mathrm{mm}}$ \\
Auxiliary damping coefficient, $\boldsymbol{c}_{\boldsymbol{r}}$ & 0.035 & $\frac{\mathrm{kN}}{\mathrm{mm} / \mathrm{sec}}$ \\
Relief force, $\boldsymbol{f}_{\boldsymbol{r}}$ & 25.5 & $\mathrm{kN}$ \\
Friction force, $\boldsymbol{f}_{\boldsymbol{f}}$ & 0 & $\mathrm{kN}$ \\
\hline
\end{tabular}

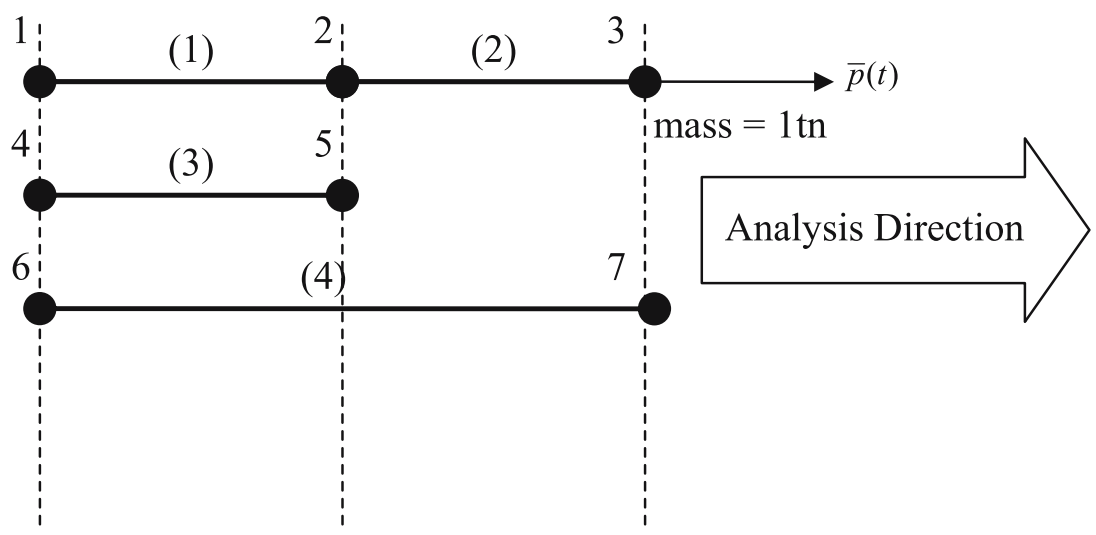

Figure 11. Analysis modelling for SAP2000 analysis procedure.

reveal that SAP2000 can accurately describe the energy-dissipating behaviour of VDHD; the simulated results almost coincide with the results of the discrete mathematical model. These outcomes verify that SAP2000 is feasible for analysing the component behaviour of VDHD.

Table 2. The element parameters for analysis modelling of SAP2000.

\begin{tabular}{|c|c|c|}
\hline Element no. & Element type & Parameters \\
\hline 1 & Plastic & $\begin{array}{l}K=255 \mathrm{kN} / \mathrm{mm} \\
F_{y}=25 \cdot 5 \mathrm{kN} \\
K_{p}=0 \\
\text { Exponential }=20\end{array}$ \\
\hline 2 & Damper & $\begin{array}{l}K=14 \mathrm{kN} / \mathrm{mm} \\
C=1.98 \mathrm{kNsec} / \mathrm{mm}\end{array}$ \\
\hline 3 & Damper & $\begin{array}{l}K=100 \mathrm{kN} / \mathrm{mm} \\
C=0.035 \mathrm{kNsec} / \mathrm{mm}\end{array}$ \\
\hline 4 & Plastic (linear) & $K=1000000 \mathrm{kN} / \mathrm{mm}$ \\
\hline
\end{tabular}




\subsection{Discussion}

There are some obvious differences between the experimental results and these two simulated results as shown in figures $12 \mathrm{e}$ and $\mathrm{f}$. The insufficient model pressure of hydraulic cylinder causes negative pressure on the backpressure side when the component test is carried out using high frequency reciprocating test. This phenomenon of negative-pressure brings about displacement sliding clearance that it is too late to raise the resistant force in reverse motion. Such an experimental design glitch cannot be considered as defects of the analytical model. The experimental value will return to the full situation of hysteretic loop that is similar to the analytical results under sufficient model pressure of hydraulic cylinder. On the other hand, the simulated results using SAP2000 and the discrete solution completely match with each other

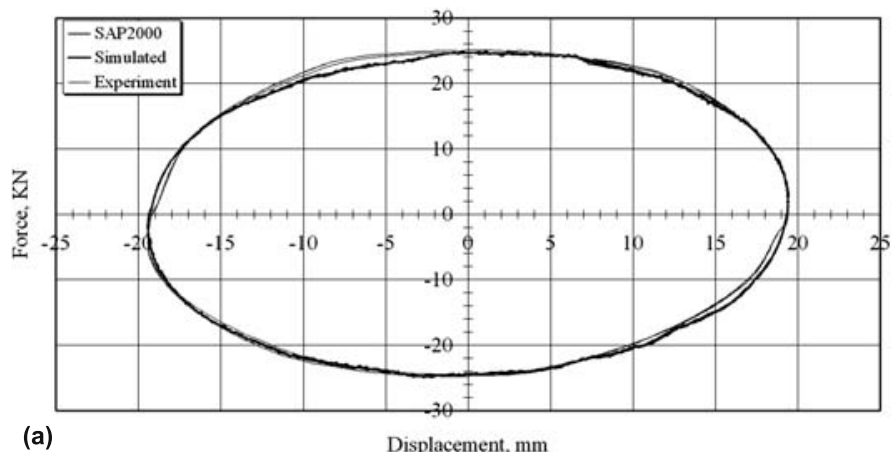

(a)

Displacement, mm

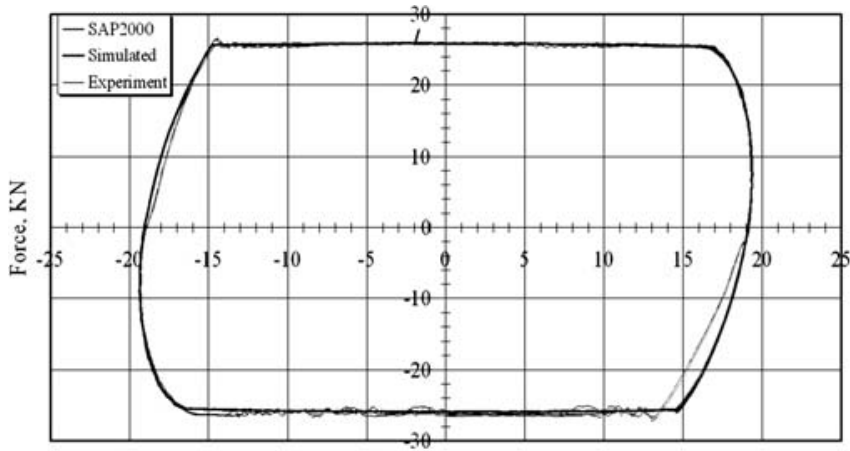

(b)

Displacement, mm

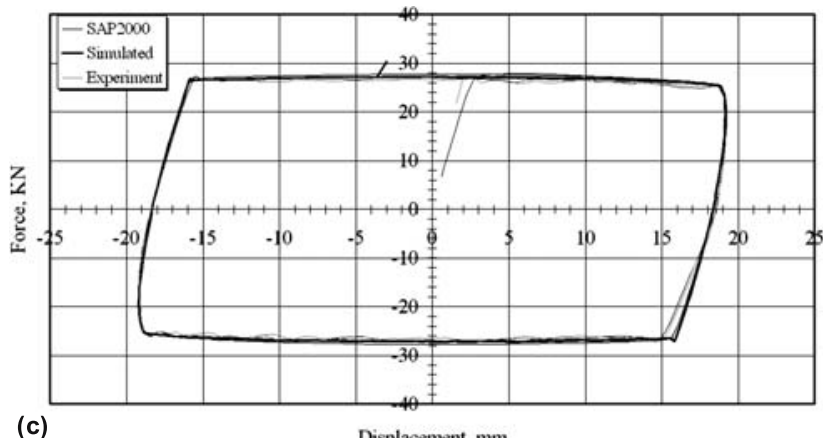

(c)

Displacement. mm

Figure 12. (Continued). 

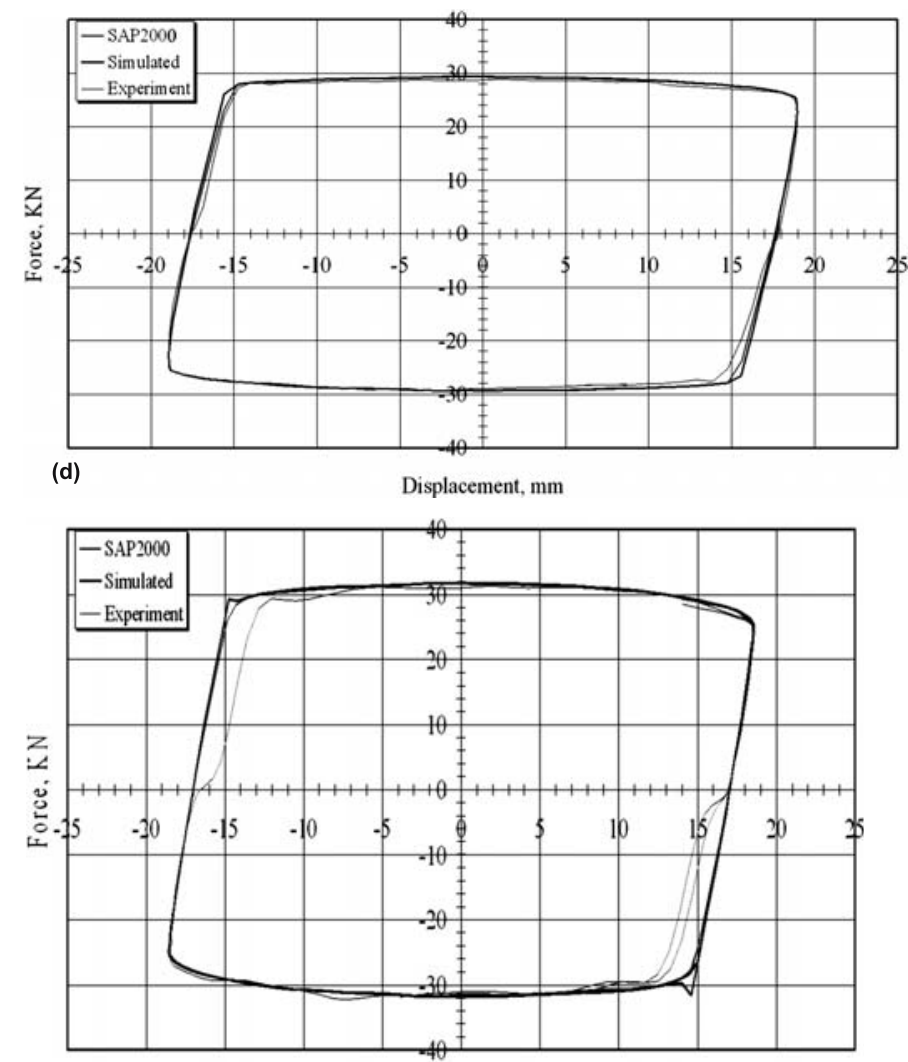

(e)

Displacement, mm

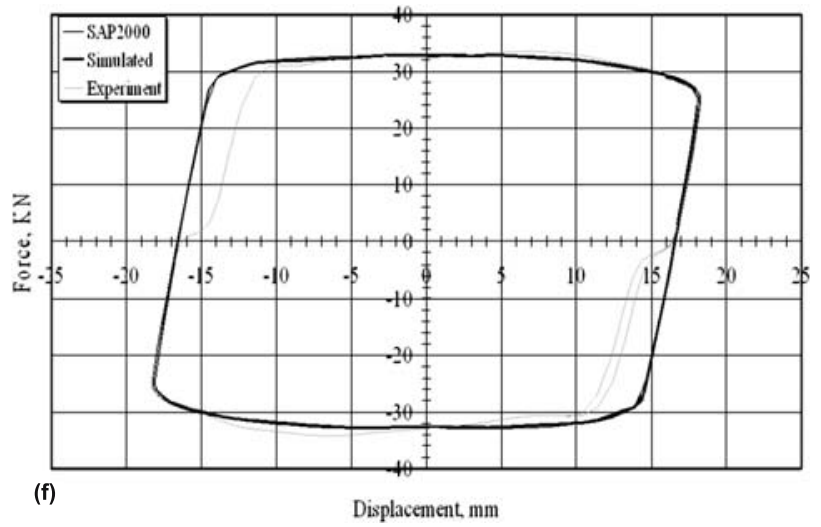

Figure 12. (a) Amplitude $=20 \mathrm{~mm}$, Frequency $=0.1 \mathrm{~Hz}$; (b) Amplitude $=20 \mathrm{~mm}$, Frequency $=0.2 \mathrm{~Hz}$; (c) Amplitude $=20 \mathrm{~mm}$, Frequency $=0.5 \mathrm{~Hz} ;$ (d) Amplitude $=20 \mathrm{~mm}$, Frequency $=1.0 \mathrm{~Hz} ;(\mathbf{e})$ Amplitude $=20 \mathrm{~mm}$, Frequency $=1.6 \mathrm{~Hz} ;(\mathbf{f})$ Amplitude $=20 \mathrm{~mm}$, Frequency $=2 \cdot 0 \mathrm{~Hz}$.

even under conditions of high frequency. These results demonstrate that the dynamic analysis program with linear viscous damping and elastic plastic element can be used for evaluating the seismic proof performance of structures with VDHD installed. 
Table 3. The relationship of loading frequency, length of sub-step and analysis error.

\begin{tabular}{lcc}
\hline Loading frequency $(\mathrm{Hz})$ & Length of sub-step $(\mathrm{sec})$ & Error $(\%)$ \\
\hline $0 \cdot 1$ & $0 \cdot 01$ & 0.53 \\
$0 \cdot 2$ & $0 \cdot 00002$ & 0.77 \\
$0 \cdot 5$ & 0.00001 & 1.43 \\
$1 \cdot 0$ & 0.000005 & 1.02 \\
$1 \cdot 6$ & 0.000005 & 1.25 \\
$2 \cdot 0$ & 0.000002 & $1 \cdot 31$ \\
\hline
\end{tabular}

\subsection{Suggestion}

Although the results present in this paper verify the practicability of nonlinear link of SAP2000 to analyse the energy-dissipating behaviour of VDHD, keeping the equilibrium of internal node 2 is difficult when the elastic plastic element of simulated friction element enters the plastic stage. Therefore, the analysis sub-step length must be substantially reduced to attain the equilibrium of internal node. Table 3 lists the relationship of loading frequency and analysis error with the length of sub-step; the analysis results show that analysis errors are below $2 \%$. Hence, in order to improve the efficiency of program analysis capability, the discrete solution as proposed (Sung et al 2007) on the length of sub-step must be set-up in the analysis procedure.

\section{Conclusions}

Results of numerical and experimental analyses show that the relationship between the force-displacement during the course of the energy dissipation of the damper can be adequately simulated using the mathematical and numerical model proposed in this study. The mathematical model combined with virtual mass joints is comparable to commercial analysis programs such as SAP2000N and other similar programs as demonstrated by the data presented in this paper. Although adding a simulated element to the main program of commercial analysis programs is impossible, the model can be applied in the nonlinear link to use its analytical program for simulating similar energy-dissipating behaviour. Therefore, this research has considerable value on the engineering design and calculation analysis of the damper proposed in this research.

We gratefully acknowledge the National Science Council of Taiwan for supporting this research through grant No. NSC 97-2626-M-167-001.

\section{References}

Constantinou M C, Soong T T, Dargush G F 1998 Passive energy dissipation systems for structural design and retrofit. Multidisciplinary center for earthquake engineering research, Monograph, NY, U.S.A. No. 1: $72-74$ 
Dyke S J, Spencer Jr B F, Sain M K, Carlson J D 1998 An experimental study of MR dampers for seismic protection. Smart Master Struct. 7: 693-703

Hsu D S, Ho C Y, Lee Y F, Chiou S I 2000 Study on the high frequency characteristics of fluid damper. J. Struct. Eng. 15(3): 3-19

Inaudi J A, Hayen J C 1995 Research on variable-structure system in the United States. International Post-Smirt Conference Seminar on Seiesmic Isolation, Passive Energy Dissipation and Control of Vibrations of Structures, Santiago, Chile

Iwan W D, Wang L J 1998 A comparison of control algorithms for active interaction control of civil engineering. Proceedings of $2^{\text {nd }}$ World conference on structural control Kyoto, Japan 2: 1559-1566

Kurata N, Kobori T, Takahashi M, Niwa N, Midorikawa H 1999 Actual seismic response controlled building with semi-active damper system. Earthquake Eng. and Struct. Dynamics 28: 1427-1447

Kurata N, Kobori T, Takaashi M, Ishibashi T, Niwa N, Tagami J, Midorikawa H 2000 Forced vibration test of a building with semi-active damper system. Earthquake Engineering and Structural Dynamics 29: 629-645

Laursen T A, Oancea V G 1997 On the constitutive modelling and finite element computation of ratedependent frictional sliding in large deformations. Computer Methods in Applied Mech. and Eng. 143: 197-227

Lee D, Taylor D P 2001 Viscous damper development and future trends. The Structural design of Tall Buildings 10: 311-320

Liu W N, Meschke G, Mang H A 2000 On the approximations of the tangential slip in frictional contact analyses. Computers and Structures 78: 53-62

Makris N, Constantinou M C 1991 Fractional_derivative maxwell model for viscous dampers. J. Struct. Eng. 122(5): 485-493

Makris N, Constantinou M C 1993 Models of viscoelasticity with complex order derivatives. J. Eng. Mech. 119(7): 1453-1464

Makris N, Dargush G F, Constantinou M C 1995 Dynamic analysis of viscoelastic fluid dampers. J. Eng. Mech. 121(10): 1114-1121

Patten W N, Sack R L, Qiwei H 1996 Controlled semiactive hydraulic vibration absorber for bridges. J. Struct. Eng. 122(2): 187-192

SAP2000 Linear and nonlinear static and dynamic analysis and design of three-dimensional structure. Vol. 8.0. Computers and structures Inc., Berkeley, California, USA2002

Shih M H, Sung W P, Go C G 2002 Development of accumulated semi-active hydraulic damper. Experimental Techniques 26(5): 29-32

Shih M H, Sung W P, Go C G 2002 Study on the design and performance of displacement dependent hydraulic damper. J. Struct. Eng. 17(1): 81-90

Shih M H, Sung W P, Go C G A 2003 Design concept with a displacement dependent semi-active hydraulic damper for energy-dissipating. Experimental Techniques 27(6): 53-56

Shih M H, Sung W P 2004 The energy-dissipating behaviour of displacement dependent semi-active hydraulic damper. J. Struct. Mechanics and Earthquake Eng. Japan Society of Civil Engineering, 21(2): $121 \mathrm{~s}-129 \mathrm{~s}$

Shih M H, Sung W P 2006 Development and seismic reduction performance of velocity and displacement dependent hydraulic damper. Experimental Techniques 30(3): 41-45

Sivaselvan M V, Reinhorm A M 2000 Hysteretic models for deteriorating inelastic structures. J. Eng. Mech. 126: 633-640

Sung W P, Shih M H, Tung S H 2007 Mathematical modelling for energy dissipation behaviour of velocity dependent hydraulic damper. J. Civil Eng. Res. and Practice 4(2): 15-29

Symans M D, Constantinou M C 1997 Seismic testing of a building structure with a semi-active fluid damper control system. Earthquake Eng. and Struct. Dynamics 26: 759-777 
Taylor D P 1992 Fluid dampers for applications of seismic energy-dissipating and seismic isolation. Eleventh World Conference on Earthquake Engineering Research, State University of New York at Buffalo, No. 798

Xu Y X, Qu W L, Ko J M 2000 Seismic response control of frame structures using magnetorheological/electrorheological dampers. Eng. and Struct. Dynamics 29: 557-575

Yang J N, Kim J H, Agrawal A K 2000 Resetting semiactive stiffness damper for seismic response control. J. Struct. Eng. 126(12): 1427-1432 